\title{
ALGEBRAIC CHARACTERIZATIONS OF MEASURE ALGEBRAS
}

\author{
THOMAS JECH
}

(Communicated by David Preiss)

\begin{abstract}
We present necessary and sufficient conditions for the existence of a countably additive measure on a Boolean $\sigma$-algebra. For instance, a Boolean $\sigma$-algebra $B$ is a measure algebra if and only if $B-\{\mathbf{0}\}$ is the union of a chain of sets $C_{1} \subset C_{2} \subset \ldots$ such that for every $n$,

(i) every antichain in $C_{n}$ has at most $K(n)$ elements (for some integer $K(n)$ ),

(ii) if $\left\{a_{n}\right\}_{n}$ is a sequence with $a_{n} \notin C_{n}$ for each $n$, then $\lim _{n} a_{n}=\mathbf{0}$, and

(iii) for every $k$, if $\left\{a_{n}\right\}_{n}$ is a sequence with $\lim _{n} a_{n}=\mathbf{0}$, then for eventually all $n, a_{n} \notin C_{k}$.

The chain $\left\{C_{n}\right\}$ is essentially unique.
\end{abstract}

\section{Statement of Results}

A Boolean algebra is an algebra $B$ of subsets of a given nonempty set $X$, with Boolean operations $a \cup b, a \cap b,-a=X-a$, and the zero and unit elements $\mathbf{0}=\emptyset$ and $\mathbf{1}=X$. A Boolean $\sigma$-algebra is a Boolean algebra $B$ such that every countable set $A \subset B$ has a supremum $\sup A=\bigvee A$ (and an infimum $\inf A=\bigwedge A$ ) in the partial ordering of $B$ by inclusion.

Definition 1.1. A measure (more precisely, a strictly positive $\sigma$-additive probability measure) on a Boolean $\sigma$-algebra $B$ is a real valued function $m$ on $B$ such that

(i) $m(\mathbf{0})=0, m(a)>0$ for $a \neq \mathbf{0}$, and $m(\mathbf{1})=1$,

(ii) $m(a) \leq m(b)$ if $a \subset b$,

(iii) $m(a \cup b)=m(a)+m(b)$ if $a \cap b=\mathbf{0}$,

(iv) $m\left(\bigvee_{n=1}^{\infty} a_{n}\right)=\sum_{n=1}^{\infty} m\left(a_{n}\right)$ whenever the $a_{n}$ are pairwise disjoint.

A measure algebra is a Boolean $\sigma$-algebra that carries a measure.

Let $B$ be a Boolean algebra and let $B^{+}=B-\{\mathbf{0}\}$. A set $A \subset B^{+}$is an antichain if $a \cap b=\mathbf{0}$ whenever $a$ and $b$ are distinct elements of $A$. A partition $W$ (of $\mathbf{1}$ ) is a maximal antichain, i.e. an antichain with $\bigvee W=1$. B satisfies the countable chain condition (ccc) if it has no uncountable antichains. $B$ is weakly distributive if for every sequence $\left\{W_{n}\right\}_{n}$ of partitions there exists a partition $W$ with the property that each $a \in W$ meets only finitely many elements of each $W_{n}$.

If $B$ is a measure algebra, then $B$ satisfies the ccc and is weakly distributive. Below we present additional, purely algebraic, conditions that characterize measure algebras.

Received by the editors December 11, 2006.

2000 Mathematics Subject Classification. Primary 28A60, 06E10.

This work was supported in part by GAAV Grant IAA100190509. 
If $\left\{a_{n}\right\}_{n}$ is a sequence in a Boolean $\sigma$-algebra $B$, one defines

$$
\limsup _{n} a_{n}=\bigwedge_{n=1}^{\infty} \bigvee_{k=n}^{\infty} a_{k} \text { and } \liminf _{n} a_{n}=\bigvee_{n=1}^{\infty} \bigwedge_{k=n}^{\infty} a_{k},
$$

and if $\limsup _{n} a_{n}=\liminf _{n} a_{n}=a$, then $a$ is the limit of the sequence, denoted $\lim _{n} a_{n}$.

Theorem 1.2. A Boolean $\sigma$-algebra $B$ is a measure algebra if and only if it is weakly distributive and $B^{+}$is the union of a countable family $\left\{C_{n}\right\}_{n}$ such that for every $n$,

(i) every antichain in $C_{n}$ has at most $K(n)$ elements (for some integer $K(n)$ ), and

(ii) if $\left\{a_{n}\right\}_{n}$ is a sequence with $a_{n} \notin C_{n}$ for each $n$, then $\lim _{n} a_{n}=\mathbf{0}$.

Theorem 1.3. A Boolean $\sigma$-algebra $B$ is a measure algebra if and only if $B^{+}$is the union of a countable family $\left\{C_{n}\right\}_{n}$ such that for every $n$,

(i) every antichain in $C_{n}$ has at most $K(n)$ elements (for some integer $K(n)$ ),

(ii) if $\left\{a_{n}\right\}_{n}$ is a sequence with $a_{n} \notin C_{n}$ for each $n$, then $\lim _{n} a_{n}=\mathbf{0}$, and

(iii) for every $k$, if $\left\{a_{n}\right\}_{n}$ is a sequence with $\lim _{n} a_{n}=\mathbf{0}$, then for eventually all $n, a_{n} \notin C_{k}$.

Theorem 1.4. A Boolean $\sigma$-algebra $B$ is a measure algebra if and only if it is weakly distributive and $B^{+}$is the union of a countable family $\left\{C_{n}\right\}_{n}$ such that for every $n$,

(i) every antichain in $C_{n}$ has at most $K(n)$ elements, and

(ii) for every $n$ and all $a$ and $b$, if $a \cup b \in C_{n}$, then either $a \in C_{n+1}$ or $b \in C_{n+1}$

If a Boolean $\sigma$-algebra $B$ satisfies the ccc, then weak distributivity is equivalent to this condition: if $\left\{W_{n}\right\}_{n}$ is a sequence of partitions, then each $W_{n}$ has a finite subset $E_{n}$ such that $\lim _{n} \bigcup E_{n}=\mathbf{1}$.

Definition 1.5. A Boolean $\sigma$-algebra $B$ is uniformly weakly distributive if there exists a sequence of functions $\left\{F_{n}\right\}_{n}$ such that for each partition $W, F_{n}(W)$ is a finite subset of $W$, and if $\left\{W_{n}\right\}_{n}$ is a sequence of countable partitions, then $\lim _{n} \bigcup F_{n}\left(W_{n}\right)=\mathbf{1}$

Definition 1.6. Let $B$ be a Boolean $\sigma$-algebra. $B$ is concentrated if for every sequence $A_{n}$ of finite antichains with $\left|A_{n}\right| \geq 2^{n}$ there exist $a_{n} \in A_{n}$ such that $\lim _{n} a_{n}=\mathbf{0}$. $B$ is uniformly concentrated if there exists a function $F$ such that for each finite antichain $A, F(A)$ is an element of $A$, and if $A_{n}$ is a sequence of finite antichains with $\left|A_{n}\right| \geq 2^{n}$, then $\lim _{n} F\left(A_{n}\right)=\mathbf{0}$.

Theorem 1.7. A Boolean $\sigma$-algebra $B$ is a measure algebra if and only if it is uniformly weakly distributive and concentrated.

Theorem 1.8. A Boolean $\sigma$-algebra $B$ is a measure algebra if and only if it is weakly distributive and uniformly concentrated.

\section{BACKGROUND AND DEFINITIONS}

We give a brief history of the problem, introduce relevant definitions and state the known results. For an additional reference and a more detailed history, see [14] (in particular Fremlin's article [6]) and [4]. 
The problem of an algebraic characterization of measure algebras originated with John von Neumann. In 1937 (Problem 163 in [13]) he stated that measure algebras satisfy the ccc and are weakly distributive, and he asked if these conditions are sufficient for the existence of a measure.

In [12], Dorothy Maharam investigated Boolean $\sigma$-algebras that carry a continuous submeasure and presented necessary and sufficient conditions for the existence of such a submeasure as well as of a measure.

Definition 2.1. A (strictly positive) submeasure on a Boolean algebra $B$ is a real valued function $m$ on $B$ such that

(i) $m(\mathbf{0})=0, m(a)>0$ for $a \neq \mathbf{0}$, and $m(\mathbf{1})=1$,

(ii) $m(a) \leq m(b)$ if $a \subset b$,

(iii) $m(a \cup b) \leq m(a)+m(b)$.

A Maharam submeasure on a Boolean $\sigma$-algebra is a submeasure that is continuous:

(iv) if $\left\{a_{n}\right\}_{n}$ is a decreasing sequence in $B$ with $\bigwedge_{n=1}^{\infty} a_{n}=\mathbf{0}$, then $\lim _{n} m\left(a_{n}\right)=$ 0 .

A Maharam algebra is a Boolean $\sigma$-algebra that carries a Maharam submeasure.

A measure is a Maharam submeasure, and every Maharam algebra is ccc and weakly distributive. Maharam asked if every Maharam algebra is a measure algebra. She also proved that a Suslin line, if it exists, provides an example of a Boolean $\sigma$-algebra that is ccc and weakly distributive but not a Maharam algebra. (The existence of a Suslin line is consistent with the axioms of set theory [17], [8], but not provable in $\mathrm{ZFC}$, [15].)

In [11], John Kelley gave a combinatorial characterization of Boolean algebras that carry a finitely additive measure. A finitely additive measure on a Boolean algebra is a function $m$ that satisfies conditions (i), (ii) and (iii) of Definition 1.1. He also proved the following theorem (due independently to Pinsker [10]):

Theorem 2.2. A Boolean $\sigma$-algebra $B$ carries a measure if and only if it is weakly distributive and carries a finitely additive measure.

Proof. Let $m$ be a finitely additive measure on $B$. For every partition $W$ let $\lambda_{W}$ be the function

$$
\lambda_{W}(b)=\sup \{m(b \cap \bigcup E): E \text { is a finite subset of } W\},
$$

and let

$$
\mu(b)=\inf \left\{\lambda_{W}(b): W \text { is a partition }\right\} .
$$

The function $\mu$ is $\sigma$-additive, and weak distributivity implies that $\mu(b)>0$ for every $b \neq \mathbf{0}$.

A major advance toward the solution of Maharam's problem was the following result of Nigel Kalton and James Roberts.

Definition 2.3. A submeasure $m$ on a Boolean algebra $B$ is exhaustive if $\lim _{n} m\left(a_{n}\right)=0$ for every infinite antichain $A=\left\{a_{n}: n=1,2, \ldots\right\}$. It is uniformly exhaustive if for every $\varepsilon>0$ there exists some $n$ such that there is no sequence of $n$ disjoint elements $a_{1}, \ldots, a_{n} \in B$ with $m\left(a_{i}\right) \geq \varepsilon$ for all $i=1, \ldots, n$.

Note that a Maharam submeasure is exhaustive while a finitely additive measure is uniformly exhaustive. 
Theorem 2.4 (Kalton-Roberts [9]). If a Boolean algebra B carries a uniformly exhaustive submeasure, then $B$ carries a finitely additive measure.

Corollary 2.5. If a Boolean $\sigma$-algebra carries a uniformly exhaustive Maharam submeasure, then it is a measure algebra.

The use of the order sequential topology on $B$ (introduced by Maharam and developed by Bohuslav Balcar) resulted in further characterizations of Maharam algebras; cf. [1] and [2], in particular:

Theorem 2.6 (Balcar-Jech-Pazák [2]). A Boolean $\sigma$-algebra B carries a Maharam submeasure if and only if it is ccc and weakly distributive and has the $G_{\delta}$ property, i.e. there exists a countable family $\left\{U_{n}\right\}_{n}$ of subsets of $B$ with $\bigcap_{n=1}^{\infty} U_{n}=\{\mathbf{0}\}$ such that for every sequence $\left\{a_{k}\right\}_{k}$ with limit $\mathbf{0}$, eventually all $a_{k}$ are in $U_{n}$.

Theorem 2.6 combined with an earlier result of Todorcevic [18] shows that it is consistent that a Boolean $\sigma$-algebra is a Maharam algebra if and only if it satisfies ccc and is weakly distributive.

In [16], Michel Talagrand solved Maharam's problem by constructing a submeasure on a countable Boolean algebra that is exhaustive but not uniformly exhaustive. In view of [9] this yields a (countably generated) Maharam algebra that is not a measure algebra.

The Kalton-Roberts theorem and the Balcar-Jech-Pazák theorem are the tools we use in the proofs of Theorems 1.2-1.8.

\section{Proof of Theorems $1.2-1.8$}

First we verify that measure algebras satisfy the conditions stated in the theorems. Let $m$ be a measure on a Boolean $\sigma$-algebra $B$. $B$ is weakly distributive, in fact uniformly weakly distributive: For each $n$ and every partition $W$, let $F_{n}(W)$ be a finite subset $E$ of $W$ such that $m(\bigcup E) \geq 1-\frac{1}{2^{n}}$. If $\left\{W_{n}\right\}_{n}$ is a sequence of partitions and if $a_{n}=-\bigcup F_{n}\left(W_{n}\right)$, then we have $m\left(a_{n}\right) \leq \frac{1}{2^{n}}$ and so $\limsup _{n} a_{n}=\mathbf{0}$. Hence $\lim _{n} \bigcup F_{n}\left(W_{n}\right)=\mathbf{1}$.

For each $n$ let $C_{n}$ be the family of all $a \in B$ such that $m(a) \geq \frac{1}{2^{n}}$. We have $\bigcup_{n=1}^{\infty} C_{n}=B^{+}$, for every $n$ every antichain in $C_{n}$ has at most $2^{n}$ elements, and if $a \cup b \in C_{n}$, then either $a \in C_{n+1}$ or $b \in C_{n+1}$. If $a_{n} \notin C_{n}$ for every $n$, then $\lim _{n} a_{n}=\mathbf{0}$.

For every finite antichain $A$ let $F(A)=a \in A$ be such that $m(a) \leq m(x)$ for all $x \in A$. We have $m(F(A)) \leq \frac{1}{|A|}$, and so if $\left\{A_{n}\right\}_{n}$ is a sequence of finite antichains with $\left|A_{n}\right| \geq 2^{n}$, then for each $n, m\left(F\left(A_{n}\right)\right) \leq \frac{1}{\left|2^{n}\right|}$ and it follows that $\lim _{n} F\left(A_{n}\right)=\mathbf{0}$.

We shall prove that the conditions in Theorems $1.2-1.8$ imply the existence of a measure.

Lemma 3.1. Let $B$ be a weakly distributive Boolean $\sigma$-algebra that satisfies the conditions of Theorem 1.2. Then $B$ is uniformly weakly distributive.

Proof. Let $\left\{C_{n}\right\}_{n}$ be a countable family that has properties (i) and (ii). Without loss of generality we may assume that each $C_{n}$ is upward closed, i.e. if $a \subset b$ and $a \in C_{n}$, then $b \in C_{n}$. To begin with, condition (i) implies ccc and so every antichain is at most countable. Let $W$ be a partition and $n$ a number. We shall define $F_{n}(W)$ so that the functions $F_{n}$ witness uniform weak distributivity. 
We claim that there exists a finite set $E \subset W$ (possibly empty) such that there exists no nonempty finite set $F \subset W-E$ with $\bigcup F \in C_{n}$. If not, then we can find an infinite sequence $\left\{E_{k}\right\}_{k}$ of disjoint finite subsets of $W$ producing an infinite antichain $\left\{\bigcup E_{k}: k=1,2, \ldots\right\}$ in $C_{n}$. We let $F_{n}(W)$ be such an $E$.

Now let $\left\{W_{n}\right\}_{n}$ be a sequence of partitions. Since $B$ is weakly distributive there exist finite sets $E_{n} \subset W_{n}$ such that $\lim _{n} \bigcup E_{n}=1$. For each $n$ let $a_{n}=\bigcup E_{n}-$ $\bigcup F_{n}(W)$. By the definition of $F_{n}(W)$ we have $a_{n} \notin C_{n}$ and hence $\lim _{n} a_{n}=\mathbf{0}$. It follows that $\lim _{n} \bigcup F_{n}(W)=\mathbf{1}$.

Lemma 3.2 ([4], p. 259). If $B$ is a uniformly weakly distributive ccc Boolean $\sigma$-algebra, then $B$ has the $G_{\delta}$ property.

Proof. Let $F_{n}$ be functions that witness the uniform weak distributivity. For each $n$ we let

$$
U_{n}=\left\{a \in B: a \text { is disjoint from } \bigcup F_{n}(W) \text { for some partition } W\right\} .
$$

First we claim that $\bigcap_{n=1}^{\infty} U_{n}=\{\mathbf{0}\}$ : If $a \in U_{n}$ for each $n$, and if $W_{n}$ are partitions such that $a \cap \bigcup F_{n}\left(W_{n}\right)=\mathbf{0}$, then because $\lim _{n} \bigcup F_{n}\left(W_{n}\right)=\mathbf{1}$, we have $a=\mathbf{0}$. Now let $\left\{a_{k}\right\}_{k}$ be a sequence with limit $\mathbf{0}$, and let $n$ be an integer. There is a decreasing sequence $\left\{b_{k}\right\}_{k}$ such that $b_{1}=\mathbf{1}, a_{k} \subset b_{k}$ for each $k$ and $\bigwedge_{k=1}^{\infty} b_{k}=\mathbf{0}$. Let $W$ be the partition $\left\{b_{k+1}-b_{k}: k=1,2, \ldots\right\}$ and let $E=F_{n}(W)$. There is some $K$ such that $b_{K} \cap \bigcup E=\mathbf{0}$ and hence $a_{k} \in U_{n}$ for all $k \geq K$.

Lemma 3.3. Let $B$ be a Maharam algebra with a Maharam submeasure $m$ and assume that $B$ satisfies the conditions of Theorem 1.2. Then $m$ is uniformly exhaustive.

Proof. Let $\left\{C_{n}\right\}_{n}$ be a countable family with properties (i) and (ii). In order to verify that $m$ is uniformly exhaustive it suffices to show that for every $\varepsilon>0$ there is some $n$ such that $\{a \in B: m(a) \geq \varepsilon\} \subset C_{n}$. If not, let $\varepsilon$ be a counterexample. For each $n$ we pick $a_{n} \notin C_{n}$ with $m\left(a_{n}\right) \geq \varepsilon$. By (ii), $\lim _{n} a_{n}=\mathbf{0}$. Since $m$ is continuous, we have $\lim _{n} m\left(a_{n}\right)=0$, a contradiction.

Now Theorem 1.2 follows: If $B$ satisfies the conditions, then by Lemmas 3.1, 3.2 and the Balcar-Jech-Pazák Theorem, $B$ carries a Maharam submeasure, and by Lemma 3.3 and the Kalton-Roberts Theorem, $B$ carries a measure.

Having proved Theorem 1.2, for Theorem 1.3 it suffices to show that under the conditions of the theorem, $B$ is weakly distributive. As $B$ satisfies ccc it is enough to show that if for every $k, \lim _{n} a_{n}^{k}=\mathbf{0}$, then there is a function $n(k)$ such that $\lim _{k} a_{n(k)}^{k}=\mathbf{0}$ (see [4], p. 253). This "diagonal property" is verified using (iii) and (ii).

Turning our attention to Theorem 1.4, we will show that the conditions of Theorem 1.4 imply the conditions of Theorem 1.2. Let $B$ be a weakly distributive Boolean $\sigma$-algebra and let $\left\{C_{n}\right\}_{n}$ be a countable family that has properties (i) and (ii) of Theorem 1.4. Notice that if we replace each $C_{n}$ by the set $\left\{x \in B^{+}:(\exists y \subset x) y \in C_{1} \cup \cdots \cup C_{n}\right\}$, then the family still has properties (i) and (ii). Thus we assume that $C_{1} \subset C_{2} \subset \ldots$ and that each $C_{n}$ is upward closed. The following lemma shows that $\left\{C_{n}\right\}_{n}$ satisfies condition (ii) of Theorem 1.2.

Lemma 3.4. If $a_{n} \notin C_{n}$ for each $n$, then $\lim \sup _{n} a_{n}=\mathbf{0}$. 
Proof. Let $a=\limsup _{n} a_{n}$ and assume that $a \neq \mathbf{0}$. For each $n$ and each $k$, let $b_{n k}=a_{n+1} \vee \cdots \vee a_{n+k}$. From (ii) it follows that $b_{n k} \notin C_{n}$, for all $k$.

We have $a=\lim _{n} \lim _{k} b_{n k}$, and by weak distributivity there exists for each $n$ some $k(n)$ such that $a=\lim _{n} b_{n, k(n)}$. Since $a \neq \mathbf{0}$, there exist some $b \neq \mathbf{0}, b \subset a$ and some $N$ such that $b \subset b_{n, k(n)}$ for all $n \geq N$. Let $n \geq N$ be such that $b \in C_{n}$. Since $C_{n}$ is upward closed, we have $b_{n, k(n)} \in C_{n}$, a contradiction.

For Theorem 1.7, let $B$ be a Boolean $\sigma$-algebra that satisfies the conditions of Theorem 1.7. By Lemma $3.2 B$ has the $G_{\delta}$ property, and Theorem 2.6 shows that $B$ is a Maharam algebra as long as it is ccc. We use the following lemma:

Lemma 3.5 ([3]). If $B$ is a uniformly weakly distributive Boolean $\sigma$-algebra then $B$ satisfies the $c c c$.

Proof. Let $\bar{B}$ be the regular completion of $B$. Since $B$ is dense in $\bar{B}$, every partition in $\bar{B}$ has a refinement in $B$ and limits of sequences in $B$ are the same in $\bar{B}$ as in $B$. Hence $\bar{B}$ is uniformly weakly distributive. If $\bar{B}$ has a partition of size $\omega_{1}$, then $P\left(\omega_{1}\right)$ is a complete subalgebra of $\bar{B}$ and therefore it is uniformly weakly distributive. By [3] $P\left(\omega_{1}\right)$ is not uniformly weakly distributive and so $\bar{B}$, and hence $B$, satisfies the ccc.

Hence $B$ carries a Maharam submeasure by the Balcar-Jech-Pazák Theorem.

Lemma 3.6. Let $B$ be a Maharam algebra with a Maharam submeasure $m$ and assume that $B$ is concentrated. Then $m$ is uniformly exhaustive.

Proof. If $m$ is not uniformly exhaustive, then there exists an $\varepsilon>0$ such that for every $n$ there is an antichain $A_{n}$ of size $2^{n}$ with $m(a) \geq \varepsilon$ for all $a \in A_{n}$. This contradicts the condition that there exists a sequence $\left\{a_{n}\right\}_{n}$ such that $a_{n} \in A_{n}$ and $\lim _{n} a_{n}=\mathbf{0}$.

This completes the proof of Theorem 1.7: if $B$ satisfies its conditions, then $B$ carries a Maharam submeasure by the Balcar-Jech-Pazák Theorem, and by the Kalton-Roberts Theorem it carries a measure.

For Theorem 1.8, we will show that if $B$ satisfies the conditions of Theorem 1.8, then it satisfies the conditions of Theorem 1.2. Let $B$ be a weakly distributive Boolean $\sigma$-algebra and let $F$ be a function acting on finite antichains witnessing that $B$ is uniformly concentrated.

For each $n$ we let

$$
C_{n}=\left\{a \in B^{+}: a \neq F(A) \text { for every antichain } A \text { of size } \geq 2^{n}\right\} .
$$

If $a$ is such that $a \notin C_{n}$ for each $n$, then there exist antichains $A_{n}$ such that $\left|A_{n}\right| \geq$ $2^{n}$ and $a=F\left(A_{n}\right)$. Since $\lim _{n} F\left(A_{n}\right)=\mathbf{0}$ we have $a=\mathbf{0}$, and so $\bigcup_{n=1}^{\infty} C_{n}=B^{+}$. If $\left\{a_{n}\right\}_{n}$ is a sequence such that $a_{n} \notin C_{n}$ for each $n$, then there exist antichains $A_{n}$ such that $\left|A_{n}\right| \geq 2^{n}$ and $a_{n}=F\left(A_{n}\right)$. Hence $\lim _{n} a_{n}=\mathbf{0}$. Finally, every antichain in $C_{n}$ has fewer than $2^{n}$ elements: If $A$ is an antichain of size $\geq 2^{n}$, then $F(A) \notin C_{n}$ and so $A$ is not a subset of $C_{n}$. Hence $B$ satisfies the assumptions of Theorem 1.2.

\section{ODDS AND ENDS}

A Boolean algebra $B$ satisfies the $\sigma$-bounded $c c$ (chain condition) if $B^{+}$is the union of a countable family $\left\{C_{n}\right\}_{n}$ such that for every $n$, every antichain in $C_{n}$ has at most $K(n)$ elements (for some integer $K(n)$ ). $B$ satisfies the $\sigma$-finite $c c$ if $B^{+}$is 
the union of a countable family $\left\{C_{n}\right\}_{n}$ such that for every $n$, every antichain in $C_{n}$ if finite. These conditions were explicitly stated in [7].

The conditions in Theorems 1.2, 1.3 and 1.4 state that $B$ is $\sigma$-bounded cc but require that the $C_{n}$ have an additional property. This is necessary: Talagrand's result [16] yields a Maharam algebra that is $\sigma$-bounded cc but is not a measure algebra. In contrast, Stevo Todorcevic proved in [19] that a Boolean $\sigma$-algebra $B$ is a Maharam algebra if and only if it is weakly distributive and $\sigma$-finite cc. Notice that if $B$ carries a Maharam submeasure $m$, then $B^{+}=\bigcup_{n=1}^{\infty} C_{n}$ such that $\left\{C_{n}\right\}_{n}$ witnesses the $\sigma$-finite cc and also has the additional properties from Theorems 1.2 , 1.3 and 1.4. (Let $C_{n}=\left\{a \in B: m(a) \geq \frac{1}{2^{n}}\right\}$.)

In Theorem 1.3 we may assume that the $C_{n}$ form a chain: $C_{1} \subset C_{2} \subset \ldots$ It turns out that such a chain is essentially unique:

Proposition 4.1. If $\left\{C_{n}\right\}$ and $\left\{C_{n}^{\prime}\right\}$ are two chains that satisfy conditions (ii) and (iii) of Theorem 1.3, then $\left\{C_{n}\right\}$ and $\left\{C_{n}^{\prime}\right\}$ are mutually cofinal: for every $k$ there is an $n$ such that $C_{k} \subset C_{n}^{\prime}$.

Proof. If not, then there is a sequence $\left\{a_{n}\right\}$ in $C_{k}$ such that $a_{n} \notin C_{n}^{\prime}$ for each $n$. Hence $\lim a_{n}=\mathbf{0}$, a contradiction.

Condition (iii) of Theorem 1.3 is the $G_{\delta}$ property, and implies that for every $n$, every antichain in $C_{n}$ is finite; hence it implies ccc. Conditions (ii) and (iii) imply that $B$ is weakly distributive and so every $B$ that satisfies (ii) and (iii) of Theorem 1.3 is a Maharam algebra.

As David Fremlin pointed out, if we drop weak distributivity in Theorem 1.4, then we get a characterization of Boolean algebras that carry a finitely additive measure:

Theorem 4.2. A Boolean algebra $B$ carries a finitely additive measure if and only if $B^{+}$is the union of a countable family $\left\{C_{n}\right\}_{n}$ such that for every $n$,

(i) every antichain in $C_{n}$ has at most $K(n)$ elements, and

(ii) for every $n$ and all $a$ and $b$, if $a \cup b \in C_{n}$, then either $a \in C_{n+1}$ or $b \in C_{n+1}$.

Proof. The condition is clearly necessary. For the sufficiency we follow the construction in [1], p. 75: First, modify the $C_{n}$ so that each $C_{n}$ is upward closed and $C_{1} \subset C_{2} \subset C_{3} \subset \ldots$, and let $U_{n}=B-C_{n}$ for each $n$. For each number $r=\frac{1}{2^{n_{1}}}+\cdots+\frac{1}{2^{n_{k}}}$ with $n_{1}<\cdots<n_{k}$, let $V_{r}=\left\{u_{1} \cup \cdots \cup u_{k}: u_{i} \in U_{n_{i}}, i=\right.$ $1, \ldots, k\}$, and define $m(a)=\inf \left\{r: a \in V_{r}\right\}$. Condition (ii) implies that for each $a, \frac{1}{2^{n}} \leq m(a) \leq \frac{1}{2^{n-1}}$, where $n$ is the least $n$ such that $a \in C_{n}$. It follows that $m(a)>0$ whenever $a \neq \mathbf{0}$, and $m$ is a submeasure on $B$. By (i), $m$ is uniformly exhaustive, and so by the Kalton-Roberts Theorem $B$ carries a finitely additive measure.

In Definition 1.6 of (uniformly) concentrated, the assumption that $\left|A_{n}\right| \geq 2^{n}$ can be replaced by $\left|A_{n}\right| \geq \alpha_{n}$, for any sequence $\alpha_{n}$ such that $\sum_{n=1}^{\infty} \alpha_{n}<\infty$. (If $m$ is a measure, then $\sum m\left(a_{n}\right)<\infty$ implies $\lim a_{n}=\mathbf{0}$.)

Theorems 1.7 and 1.8 state that measure algebras are characterized by being uniformly weakly distributive and concentrated, resp. weakly distributive and uniformly concentrated. From Theorem 1.7 and [2] it follows that it is consistent that $B$ is a measure algebra if and only if it is ccc, weakly distributive and concentrated. On the other hand, if a Suslin tree exists, then the corresponding Suslin algebra $B$ 
is ccc, weakly distributive and concentrated, and does not carry even a Maharam submeasure:

Proposition 4.3. Let $T$ be a Suslin tree and B the corresponding complete Boolean algebra. Then $B$ is concentrated.

Proof. Let $\left\{A_{n}\right\}_{n}$ be finite antichains in $B,\left|A_{n}\right| \geq n$. We may assume that $\bigcup A_{n}=$ 1 for each $n$. A routine argument using that $T$ is a Suslin tree shows that there exists a countable family of functions $\left\{f_{k}\right\}_{k} \subset \prod_{n=1}^{\infty} A_{n}$ such that

$$
\bigvee_{k=1}^{\infty} \bigwedge_{n=1}^{\infty} f_{k}(n)=\mathbf{1}
$$

Now let $F \in \prod_{n=1}^{\infty} A_{n}$ be such that for each $n, F(n) \neq f_{i}(n)$ for all $i=1, \ldots, n$. We show that $\lim _{n} F(n)=\mathbf{0}$.

Let $a_{n}=F(n)$ and let $a=\lim \sup _{n} a_{n}$. For each $k$ let $b_{k}=\bigwedge_{n=1}^{\infty} f_{k}(n)$. Since $a_{n} \cap f_{k}(n)=\mathbf{0}$ for all $n \geq k$, we have $\bigvee_{n=k}^{\infty} a_{n} \cap b_{k}=\mathbf{0}$, and it follows that $a \cap b_{k}=\mathbf{0}$. Hence $a=\mathbf{0}$.

In the proof of Theorem 1.8 we showed that if $B$ is uniformly concentrated, then $B$ is $\sigma$-bounded cc. It turns out that a weak version of uniformly concentrated is equivalent to the $\sigma$-bounded cc, and uniformly concentrated is equivalent to conditions (i) and (ii) of Theorem 1.2:

Proposition 4.4. (a) A Boolean algebra $B$ is $\sigma$-bounded $c c$ if and only if there exists a function $F$ such that for each finite antichain $A, F(A) \in A$, and if $\left\{A_{n}\right\}_{n}$ is a sequence of finite antichains of increasing size, then $\liminf { }_{n} F\left(A_{n}\right)=\mathbf{0}$.

(b) A Boolean algebra $B$ is uniformly concentrated if and only if $B^{+}$is the union of a countable family $\left\{C_{n}\right\}_{n}$ with $C_{1} \subset C_{2} \subset \ldots$ such that for every $n$,

(i) every antichain in $C_{n}$ has fewer than $2^{n}$ elements, and

(ii) if $\left\{a_{n}\right\}_{n}$ is a sequence with $a_{n} \notin C_{n}$ for each $n$, then $\lim _{n} a_{n}=\mathbf{0}$.

Proof. (a) First assume that $B$ is $\sigma$-bounded cc, and let $\left\{C_{n}\right\}_{n}$ be a witness. We may assume that each $C_{n}$ is upward closed and that $C_{1} \subset C_{2} \subset \ldots$ If $A$ is a finite antichain, let $n$ be the least $n$ such that $A \subset C_{n}$ and let $F(A)=a \in A$ be such that $a \notin C_{n-1}$. Hence for all $N$, if $F(A) \in C_{N}$, then $A \subset C_{N}$.

Now let $\left\{A_{n}\right\}_{n}$ be a sequence of finite antichains increasing in size, and let $a_{n}=F\left(A_{n}\right)$. We claim that $\liminf _{n} a_{n}=\mathbf{0}$. If not, then there exist some $a \neq \mathbf{0}$ and some $k$ such that $a \subset a_{n}$ for all $n \geq k$. Let $N$ be such that $a \in C_{N}$; then $a_{n} \in C_{N}$ for all $n \geq k$. It follows that $A_{n} \subset C_{N}$ for all $n \geq k$, and so $C_{N}$ has antichains of arbitrary size, a contradiction.

Conversely, let $F$ be a function that satisfies the condition. If we let $C_{n}=$ $\left\{a \in B^{+}: a \neq F(A)\right.$ for every antichain of size $\left.\geq n+1\right\}$, then the same argument we used in the proof of Theorem 1.8 shows that $\bigcup_{n=1}^{\infty} C_{n}=B^{+}$and that every antichain in $C_{n}$ has at most $n$ elements.

(b) For one direction, see the proof of Theorem 1.8. For the other direction, given the $C_{n}$, we let $F(A)=a \in A$ be such that $a \notin C_{n-1}$ where $n$ is the least $n$ with $A \subset C_{n}$. Now if $\left|A_{n}\right| \geq 2^{n}$, then $A_{n} \not \subset C_{n}$ and so $F\left(A_{n}\right) \notin C_{n}$. Hence $\lim _{n} F\left(A_{n}\right)=\mathbf{0}$.

Weak distributivity has a formulation in terms of forcing: a complete ccc Boolean algebra $B$ is weakly distributive if and only if for every $B$-name $\dot{f}$ for a function 
from $\omega$ to $\omega$ there exists a function $g: \omega \rightarrow \omega$ such that

$$
\Vdash \exists N \forall n \geq N \dot{f}(n)<g(n) .
$$

(The last formula is equivalent to $\lim _{n}\|\dot{f}(n)<g(n)\|=\mathbf{1}$.)

Similarly, $B$ is concentrated if and only if for every $B$-name $\dot{f}$ for a function from $\omega$ to $\omega$ there exists a function $g: \omega \rightarrow \omega$ such that $g(n)<2^{n}$ for each $n$ and

$$
\Vdash \exists N \forall n \geq N \dot{f}(n) \neq g(n) .
$$

The following result shows that the existence of a finitely additive measure does not imply that $B$ is concentrated. The Cohen algebra carries a finitely additive measure but is not concentrated:

Proposition 4.5. The Cohen algebra is not concentrated.

Proof. We use this representation of the Cohen algebra: Let $P$ be the forcing where the forcing conditions are finite sequences $p$ of integers such that $p(n)<2^{n}$ for each $n \in \operatorname{dom}(p)$. We let $\dot{f}$ be the following name for a function from $\omega$ to $\omega$ : for each $n$ and each $k<2^{n}$ let

$$
\|\dot{f}(n)=k\|=\bigvee\{p: p(n)=k\} .
$$

Now if $g: \omega \rightarrow \omega$ is such that $g(n)<2^{n}$ for all $n$, then for every condition $p$ and every $N$ there exist a stronger condition $q$ and some $n>N$ such that $q \Vdash \dot{f}(n)=g(n)$. This shows that $\dot{f}$ is a counterexample.

Maharam algebras have a characterization in terms of infinite games. Using [1], David Fremlin proved in [5] that a strategic version of weak distributivity implies the existence of a Maharam submeasure for Boolean $\sigma$-algebras that satisfy the ccc (see [4], p. 261, for details). In [3] it is shown that the "strategic diagonal property" implies ccc. Combining this with the proof of Theorem 1.7, we obtain the following characterization of measure algebras:

Let $B$ be a complete Boolean algebra and consider the infinite game $\mathcal{G}$ in which the $n$th move of Player I is a $B$-name $\dot{f}(n)$ for an integer and the $n$th move of Player II is an integer $g(n)$. Thus I produces a $B$-name $\dot{f}$ for a function from $\omega$ to $\omega$ and II produces a function $g: \omega \rightarrow \omega$. Player II wins if

$$
\Vdash \exists N \forall n \geq N \dot{f}(n)<g(n) \text { and }\left(\dot{f}(n) \not \equiv g(n) \quad \bmod 2^{n}\right) .
$$

Theorem 4.6. A complete Boolean algebra $B$ is a measure algebra if and only if Player II has a winning strategy in the game $\mathcal{G}$.

\section{REFERENCES}

[1] B. Balcar, W. Główczyński, and T. Jech. The sequential topology on complete Boolean algebras. Fund. Math., 155(1):59-78, 1998. MR1487988 (99b:28006)

[2] B. Balcar, T. Jech, and T. Pazák. Complete ccc Boolean algebras, the order sequential topology and a problem of von Neumann. Bull. London Math. Society, 37:885-898, 2005. MR2186722 (2006j:28013)

[3] Bohuslav Balcar and Thomas Jech. Contributions to the theory of weakly distributive complete Boolean algebras. to appear.

[4] Bohuslav Balcar and Thomas Jech. Weak distributivity, a problem of von Neumann and the mystery of measurability. Bull. Symbolic Logic, 12(2):241-266, 2006. MR2223923 (2007h:03129)

[5] D. H. Fremlin. Maharam algebras. Unpublished notes.

[6] D. H. Fremlin. Measure algebras. In Handbook of Boolean algebras. Vol. 3, pages 877-980. North-Holland Publishing Co., Amsterdam, 1989. MR991611 
[7] A. Horn and A. Tarski. Measures in Boolean algebras. Trans. Amer. Math. Soc., 64:467-497, 1948. MR0028922 (10:518h)

[8] T. Jech. Non-provability of Souslin's hypothesis. Comment. Math. Univ. Carolinae, 8:291305, 1967. MR0215729 (35:6564)

[9] N. J. Kalton and J. W. Roberts. Uniformly exhaustive submeasures and nearly additive set functions. Trans. Amer. Math. Soc., 278:803-816, 1983. MR701524 (85f:28006)

[10] L. V. Kantorovič, B. Z. Vulikh, and A. G. Pinsker. Functional Analysis in Partially Ordered Spaces. 1950 (in Russian).

[11] J. L. Kelley. Measures on Boolean algebras. Pacific J. Math., 9:1165-1177, 1959. MR0108570 $(21: 7286)$

[12] D. Maharam. An algebraic characterization of measure algebras. Ann. of Math. (2), 48:154167, 1947. MR0018718 (8:321b)

[13] D. Mauldin, editor. The Scottish Book. Birkhäuser Boston, Mass., 1981.

[14] J. D. Monk, editor. Handbook of Boolean algebras. North-Holland Publishing Co., Amsterdam, 1989

[15] R. M. Solovay and S. Tennenbaum. Iterated Cohen extensions and Souslin's problem. Ann. of Math. (2), 94:201-245, 1971. MR0294139 (45:3212)

[16] M. Talagrand. Maharam's problem. Annals of Math, to appear.

[17] S. Tennenbaum. Souslin's problem. Proc. Nat. Acad. Sci. U.S.A., 59:60-63, 1968. MR0224456 $(37: 55)$

[18] S. Todorcevic. A dichotomy for P-ideals of countable sets. Fund. Math., 166(3):251-267, 2000. MR1809418 (2001k:03111)

[19] S. Todorcevic. A problem of von Neumann and Maharam about algebras supporting continuous submeasures. Fund. Math., 183:169-183, 2004. MR2127965 (2005m:28012)

Mathematical Institute, AS CR, Zitna 25, CZ - 11567 Praha 1, Czech Republic

E-mail address: jech@math.cas.cz 